\title{
Endobronchial ultrasound-guided transbronchial needle aspiration is useful in the diagnosis of lymphoma: don't give up!
}

\author{
Yilmaz A, Ozturk A \\ Atatürk Chest Diseases and Thoracic Surgery Training and Research Hospital, Interventional Pulmonology \\ Department, Ankara, Turkey. aydinyilmaz587@gmail.com
}

\section{ABSTRACT}

BACKGROUND: Endobronchial ultrasound-guided transbronchial needle aspiration (EBUS-TBNA) is considered cautiously in the diagnosis of lymphoma. In this study, we present our data with literature review, to ask the readers to re-evaluate this opinion.

MATERIALS AND METHODS: Several demographic, clinical and procedural variables of patients who had been diagnosed with lymphoma with EBUS-TBNA at our institution between January 2011 and December 2016 were noted and analyzed.

RESULTS: We analyzed 27 patients with a proven diagnosis of lymphoma, out of whom twenty patients were with classical Hodgkin's lymphoma and seven with non-Hodgkin's lymphoma. The sensitivity, specificity, positive and negative predictive values and accuracy of EBUS-TBNA for the diagnosis of lymphoma was found to be $70.3 \%, 100 \%, 100 \%, 99.4 \%$ and $99.4 \%$, respectively. The diagnostic sensitivity of EBUS-TBNA in ten patients with the history of lymphoma was $100 \%$; while in patients with no history of lymphoma it was $52.9 \%$. The patients with non-Hodgkin's lymphoma were presented at older ages $(p=0.001)$, with larger lesions $(p=$ $0.046)$ and higher SUVmax values $(p<0.001)$. No complications occurred.

CONCLUSION: EBUS-TBNA may be used as an initial diagnostic modality in patients suspicious for lymphoma instead of repeated mediastinoscopy (Tab. 2, Fig. 1, Ref. 34). Text in PDF www.elis.sk. KEY WORDS: endobronchial ultrasound, transbronchial needle aspiration, lymphoma.

\section{Introduction}

Lymphoma may present as mediastinal lymphadenopathy and isolated mediastinal mass (1). The diagnosis should be achieved by using multidirectional diagnostic modalities, including cytomorphologic studies, immunophenotyping, cytogenetic analyses, and molecular studies (2). Hence, it is recommended to obtain large biopsy specimens or excisional lymph node biopsy to provide enough material for these modalities (3-5).

Regarding the anatomical features of the mediastinum, the surgical access to the mass carries an undeniable risk of morbidity and mortality. Despite being the "gold-standard" method, mediastinoscopy, is replaced by endobronchial ultrasound-guided transbronchial needle aspiration (EBUS-TBNA) as an initial modality for the diagnosis of mediastinal lymphadenopathies and masses. As a minimally invasive technique, it has a high sensitivity and specificity for the diagnosis of lung cancer and several benign diseases such as sarcoidosis (6). Although there are encouraging results in some studies on the efficiency of EBUS-TBNA in the

Atatürk Chest Diseases and Thoracic Surgery Training and Research Hospital, Interventional Pulmonology Department, Ankara, Turkey

Address for correspondence: Aydin Yilmaz, Ataturk Chest Diseases and Thoracic Surgery Training and Research Hospital, Department of Interventional Pulmonology, Sanatoryum Cad. 06280 Kecioren, Ankara, Turkey Phone: +903125677000 diagnosis of lymphoma (7), this disease is still considered to be the “Achilles' heel" of EBUS-TBNA by many authors, as it provides a relatively smaller specimen volume for a proper diagnosis (8-10).

In their guideline for advanced diagnostic and therapeutic flexible bronchoscopy, British Thoracic Society states that currently there is not enough evidence to recommend the use of EBUS-TBNA in the diagnosis of suspected lymphoma (6). Given the paucity of the information in the literature, we decided to present our data on the use of EBUS-TBNA in the diagnosis of lymphoma, in order to expand the common knowledge for future assessments.

\section{Material and methods}

We performed a retrospective analysis of patients who underwent endobronchial ultrasound for the diagnosis of mediastinal lymph nodes and single masses adjacent to proximal airways at a single institution between January 2011 and December 2016. The patients with a proven diagnosis of lymphoma either with EBUSTBNA or subsequent surgical biopsy were involved in the study. The patients were divided into two groups, namely Hodgkin's lymphoma (HL) and non-Hodgkin's lymphoma (NHL), and analyzed accordingly. Relevant demographic characteristics, past medical history of lymphoma and some imaging features were noted. The size of the lesion and the maximum standardized uptake value (SUVmax) were measured on computed tomography (CT) and 
Tab. 1. Several comparative characteristics of the patients with lymphoma.

\begin{tabular}{|c|c|c|c|c|}
\hline & All cases $(n: 27)$ & HL (n:20) & NHL (n:7) & $\mathrm{p}^{*}$ \\
\hline Age - mean \pm SD (min-max) & $43.8 \pm 19.3(16-75)$ & $37.3 \pm 17.8(16-75)$ & $62.7 \pm 6.8(55-72)$ & 0.001 \\
\hline Sex - M:F & $18: 9$ & $12: 8$ & $6: 1$ & 0.224 \\
\hline Previous lymphoma diagnosis & 10 & 9 & 1 & 0.161 \\
\hline Size - mean $\pm S D(\min -\max )$ & $25.0 \pm 11.1(6.8-50.0)$ & $22.5 \pm 10.1(6.8-40.0)$ & $32.2 \pm 11.2(20.0-50.0)$ & 0.046 \\
\hline $\mathrm{SUVmax}-\operatorname{mean} \pm \mathrm{SD}(\min -\max )$ & $13.3 \pm 7.6(2.9-37.9)$ & $9.9 \pm 3.6(2.94-16.0)$ & $21.3 \pm 10.1(5.8-37.9)$ & $<0.001$ \\
\hline
\end{tabular}

F - female; HL - Hodgkin lymphoma; M:male; min - minimum; max - maximum; NHL - non-Hodgkin lymphoma; SD - standard deviation; SUVmax - maximum standardized uptake value; * comparisons were made between HL and NHL

positron emission tomography-computed tomography (PET-CT) fusion images, respectively.

The EBUS-TBNA procedure and specimen processing were described elsewhere (11). However, it should be re-noted that we used 22-gauge needles to sample the lymph nodes and masses. Cell blocks were prepared for every patient. Rapid on-site evaluation was not available at our center. In patients suspected of having HL or NHL, further immunohistochemical analyses were done with CD30, CD15, CD3, CD20 and CD20, CD3, CD79a, and TdT, respectively. The diagnoses were done according to World Health Organization classification. (2) We did not perform flow cytometry and fluorescent in-situ hybridization (FISH) analysis routinely at our center. All cases with a negative result in EBUS-TBNA, have undergone surgical confirmation.

IBM SPSS Statistics 20.0 was used for statistical analyses. Descriptive statistics were given as mean \pm standard deviation for continuous variables and frequency (in percent) for categorical variables. Pearson chi-square test was used to determine the association between categorical variables. When comparing continuous variables, independent-samples T-test and MannWhitney $U$ test were used. The value of $p<0.05$ was assumed as statistically significant. This study was approved by Ethical
Committee of our institution and informed consent was obtained from every patient.

\section{Results}

Among 1,471 cases who underwent EBUS-TBNA for isolated mediastinal masses and/or lymphadenopathy, twenty-seven patients $(1.8 \%)$ with a proven diagnosis of lymphoma were involved in the study. Twenty patients were with classical HL (74.0\%) and seven $(26.0 \%)$ with NHL. All NHL patients were diagnosed with diffuse B cell lymphoma. Ten patients $(37.0 \%)$ had the history of lymphoma with a suspicion of relapsed disease. In average, 1.96 lymph nodes and masses were sampled for every patient. The median number of passes per lymph node/mass was 4 (3-7).

The sensitivity, specificity, positive and negative predictive values and accuracy of EBUS-TBNA for the diagnosis of lymphoma was found to be $70.3 \%, 100 \%, 100 \%, 99.4 \%$ and 99.4 $\%$, respectively. The diagnostic sensitivity of EBUS-TBNA in ten patients with the history of lymphoma, was $100 \%$; nine of them with HL and one with NHL. The sensitivity dropped to $52.9 \%$ in patients without any history of lymphoma. The difference was statistically significant $(\mathrm{p}=0.011)$. EBUS-TBNA was diagnos-

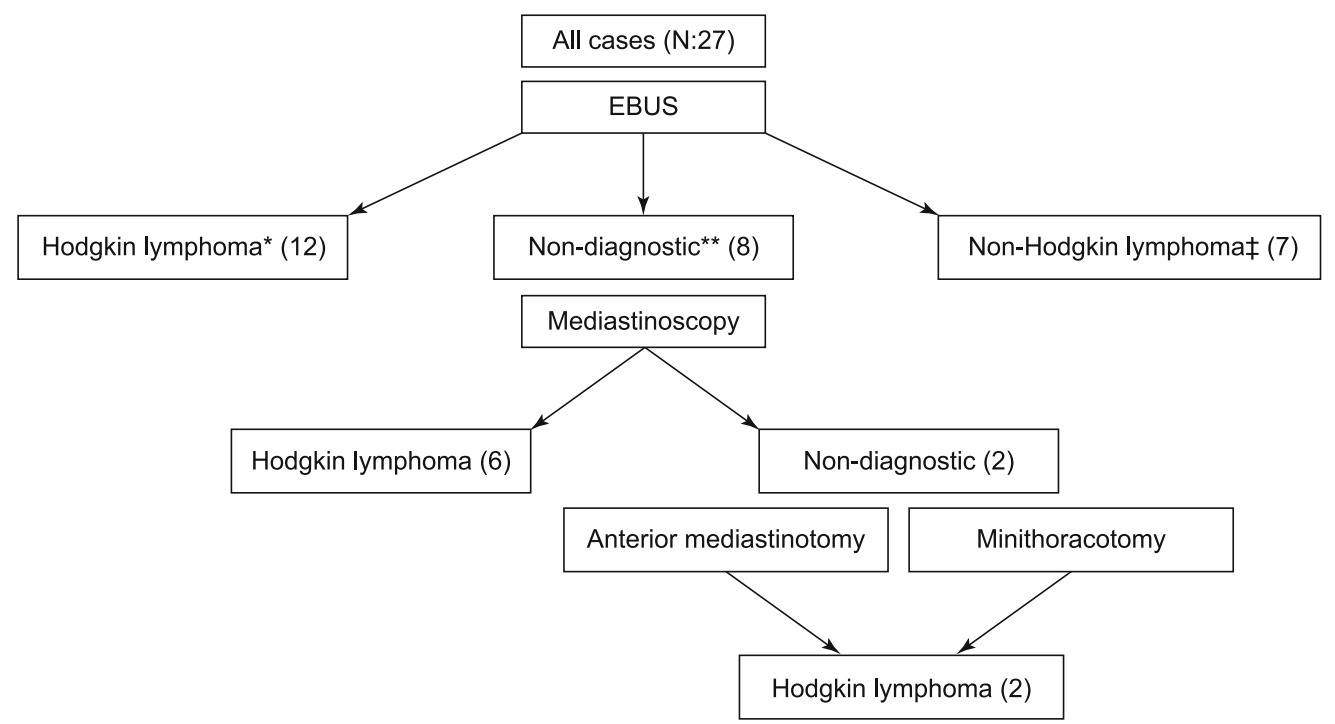

Fig. 1. Flowchart of patients enrolled in the study. * 9 cases with relapsed disease, ** Non-diagnostic results were lymphoproliferative disease ( 6 cases) and anthracosis ( 2 cases), $\$$ One case with relapsed disease. 


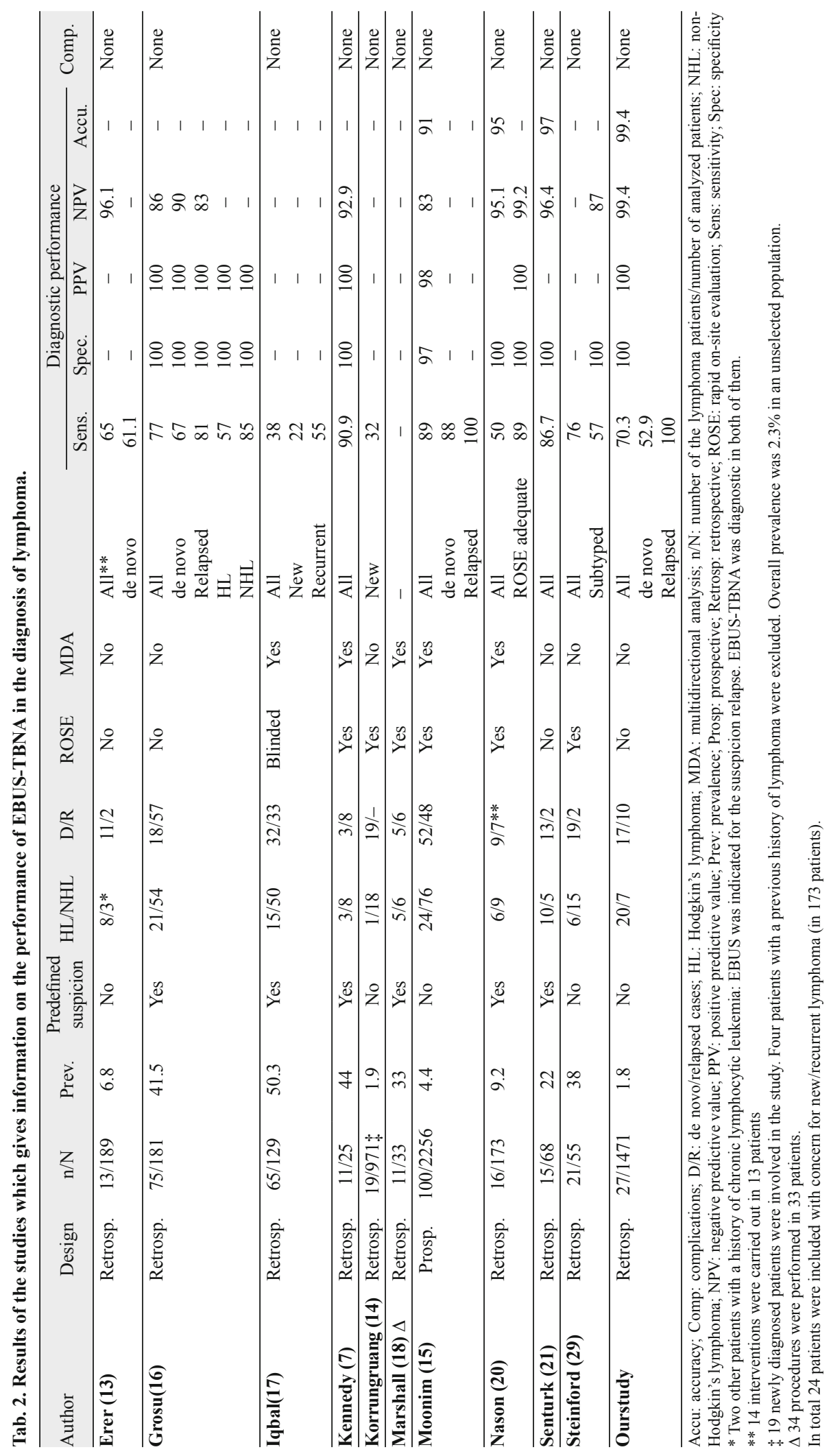

tic in all cases with NHL (100\%), but in cases with HL, the percentage was $57.8 \%(\mathrm{p}<0.001)$. The size $(\mathrm{p}=0.985)$ and SUVmax values $(\mathrm{p}$ $=0.206)$ were not statistically different between diagnosed and nondiagnosed lesions. Eight patients with a negative EBUS-TBNA have undergone mediastinoscopy, all of which was HL. Six of them were diagnosed with mediastinoscopy and two of them with subsequent anterior mediastinotomy and minithoracotomy after failed mediastinoscopy (Fig. 1).

Several characteristics of the cases are given in Table 1. The patients with NHL were presented at older ages $(p=0.001)$, with larger lesions $(p=0.046)$ and higher SUVmax values $(\mathrm{p}<0.001)$.

No complications occurred related to anesthesia and EBUS-TBNA procedure.

\section{Discussion}

The place of EBUS-TBNA in the initial diagnostic work-up of suspected lymphoma is still under debate. Many authors conclude that the existing information is scarce to establish a proper suggestion (6) while others favor the use of EBUS-TBNA regarding several promising studies (12). We think that, in general, with its capabilities and low complication rate, EBUS-TBNA should be considered in the initial management of the patients with isolated mediastinal mass and lymphadenopathy. As to the question whether it is true for the diagnosis of lymphoma, the literature lacks reliable prospective randomized studies. However, there are several retrospective analyses on the subject. We summarized the results of existing studies on the utility of EBUS-TBNA in the diagnosis of lymphoma in Table 2 and will discuss several points on different aspects of the subject.

Just as our study, many studies were retrospective in nature and searched their databases for patients 


\section{3-508}

with the final diagnosis of lymphoma. When all cases who had undergone EBUS-TBNA for isolated mediastinal mass and lymphadenopathy were included, the prevalence of lymphoma was 1.9-6.8 $\%(13-15)$. We, also, reported a similar prevalence of $1.8 \%$ in an unselected population. However, with a careful preoperative clinical assessment, a possible subgroup of patients at risk of lymphoma may be determined. In studies with data on predefined suspicion of lymphoma, the prevalence has reached 33-50.3\% (16-18). This difference is of particular importance in the management of cases suspicious for lymphoma for several reasons. First, ROSE is not routinely recommended during EBUS-TBNA operation as it does not affect the diagnostic yield (19). However, the need for ROSE is not extensively investigated in the patients with the suspicion of lymphoma. Nason et al reported that the sensitivity of EBUSTBNA has reached $89 \%$ if the samples were adequate in ROSE, while EBUS-TBNA failed to establish a proper diagnosis in 4 (25 $\%$ ) patients with inadequate samples (20). The yield may increase if ROSE is used in patients suspicious for lymphoma. Secondly, the optimal number of needle passes is not defined for this selected population. The xisting guideline recommends to perform a minimum of three passes if ROSE is not routinely used (19). However, in our experience, the lymph nodes affected with lymphoma often feel like hard rubber and a bronchoscopist usually needs more punctures to collect sufficient amount of tissue. The median number of needle passes through the studies varied between 3 to 5.2(14-16,20, $21)$. Although a pooled analysis was not performed, the sensitivity of EBUS-TBNA tended to be inferior in studies with fewer passes than in studies with a higher number of passes. In patients with a suspicion for lymphoma, more passes may be considered. Lastly, the needle property may influence the quality of specimen. Just as at our center, a 22-gauge needle is mostly used among centers. The studies showed no difference between 21-gauge and 22-gauge needles dedicated for EBUS, despite the statement that 21-gauge needles provide a better "histologic structure" $(22,23)$. However, in the current guidelines it is stated that a bronchoscopist may have preference among choices in the diagnosis of diseases that are more difficult to diagnose with EBUS-TBNA, such as lymphoma (19). Just as at our center, most of other institutions used 22-gauge aspiration needles except Iqbal et al (used 21-Gauge) (17). Inoue et al reported a case of lymphoma in whom a multidirectional analysis was done on tissue samples obtained by EBUS-TBNA. The specimen aspirated by a 22-gauge needle was sufficient for histomorphologic studies, immunohistochemistry, flow cytometry and FISH analysis (24). Additionally, Korrungruang et al used both 21- and 22-gauge needles and reported that the 21-gauge needle provided definitive diagnostic material in $75 \%$ of the patients, while the 22-gauge needle failed with $20 \%$ (14). The choices are not limited to aspiration needles. Ariza-Prota et al reported a case of anaplastic large cell lymphoma who was diagnosed with EBUSTBNA. They used a new 22-gauge EchoTip ProCore needle dedicated to EBUS-TBNA to obtain proper histologic specimen (25). The needle was compared to conventional aspiration needle used in EUS-FNA and found no difference in sense of tissue adequacy. Moreover, there was a trend toward fewer passes to obtain an adequate amount of specimen (26). Similarly, Furukawa et al used same needle with rapid on-site evaluation and reported that they obtained an adequate amount of specimen for the diagnosis of HL (27). The inferior yield of aspiration cytology is also highlighted in other studies (28). Before the suggestion can be considered final, there is a need for prospective trials directly comparing other needles to the conventional aspiration needle dedicated to EBUS.

There are several papers on the diagnostic performance of EBUS-TBNA in the diagnosis of lymphoma. Kennedy et al analyzed 25 patients with a suspicion of lymphoma. Overall sensitivity, specificity, positive and negative predictive values of EBUSTBNA in patients with suspected lymphoma were $90.9 \%, 100 \%$, $100 \%$ and $92.9 \%$, respectively. Ten patients were diagnosed with EBUS-TBNA. Flow cytometry was used when necessary. However, the procedure failed in one patient (false negative). This study is the most relevant one favoring EBUS-TBNA in the diagnosis of lymphoma. Nevertheless, it should be noted that in two of eleven patients, the findings in EBUS-TBNA was not sufficient; in one patient, mediastinoscopy was done for sub-classification; and in the other, the diagnosis had to be confirmed with bone marrow and endobronchial biopsies. Of twenty-five patients, 11 had a prior history of lymphoma in remission and EBUS-TBNA was diagnostic in all of them (eight relapses, three true negatives). However, there was one false-negative result among patients with no history of previous disease. In concordance with the literature, these data are consistent with our study. A prior history of the disease seems to be a reliable guide for the pathologist. Korrungruang et al. investigated the diagnostic utility of EBUS-TBNA only for the definitive diagnosis (with subtyping) of de novo lymphoma. They analyzed 971 procedures and found 23 patients with final diagnosis of lymphoma. Four patients with a previous history of lymphoma were excluded from the study and 19 patients remained with a newly diagnosed lymphoma. EBUS-TBNA was diagnostic in $6(32 \%)$ of 19 cases. (14) When comparing the data to other studies, the reader should note that these six patients had definitive diagnoses with sub-classification. There are ten patients with suspicion of lymphoma; four of whom remained without a subtype classification; while six have undergone further surgical biopsy. Remaining three patients were diagnosed with lymphoma only with surgical biopsy and classified as a "real" false negative. The methodology differs from other studies; Korrungruang et al. defined the diagnosis with no need for further invasive procedures. This may be of particular importance when considering the "true" sensitivity of EBUS-TBNA as a procedure with therapeutic guidance. Senturk et al. reviewed 68 cases who have undergone EBUS-TBNA with a suspicion of lymphoma. Fifteen patients were diagnosed with lymphoma while ten of them were HL. The sensitivity, specificity, negative predictive value, and diagnostic accuracy of EBUS-TBNA for the diagnosis of lymphoma were calculated as $86.7 \%, 100 \%, 96.4 \%$, and $97 \%$, respectively. They concluded that EBUS-TBNA is a useful tool in the diagnosis of lymphoma, particularly when combined with immunohistochemistry (21). Steinfort et al evaluated ninety-eight patients with isolated mediastinal lymphadenopathy and of them, twenty-one patients had the final diagnosis of lymphoma. Although EBUS has given a diagnosis of lymphoma in sixteen patients, further surgical biopsy was needed in four cases (subtyping in three and confirmation in 
one). Regarding this, the authors stated that the accurate sensitivity should be $57 \%$. Specificity and negative predictive value for detection of lymphoma were $100 \%$ and $87 \%$, respectively (29). Marshall et al analyzed 34 interventions in 33 patients with a suspicion of lymphoma. Eight cases were diagnosed with lymphoma with EBUS-TBNA (six cases of recurrent disease and two cases of new diagnosis). EBUS-TBNA was non-diagnostic in three patients; fortunately, none of them developed the disease in 17-23 months of follow-up. Two cases with a suspicion of lymphoma was diagnosed as HL with additional interventions. All EBUS-TBNA negative cases were true negatives with either reactive lymph nodes and granulomatous inflammation. There were two patients with atypical results. One of them has undergone repeated EBUSTBNA and is diagnosed with HL. Although they did not give any data on diagnostic performance, the use of EBUS-TBNA seems to be reasonable regarding the given data (18). Nason et al evaluated the patients with mediastinal lymphadenopathy to determine the ability of EBUS-TBNA with ROSE for the diagnosis of lymphoma. Of one hundred seventy-three cases, sixteen patients had the final diagnosis of lymphoma. EBUS-TBNA failed to establish a proper diagnosis in $8(50 \%)$ patients (indeterminate in three, inadequate in four and false-negative in one patient). The sensitivity was $89 \%$ when specimens were adequate for analyses. The median number of passes for each sampled node station was 3 (2-4) which was lower than in other studies (20). The only prospective study was conducted by Moonim et al. They evaluated 100 patients with de novo or suspected relapsed mediastinal lymphoma. They used immunohistochemistry, FISH, immunoglobulin heavy chain and T-cell receptor gene rearrangement studies as indicated. The sensitivity, specificity, positive and negative predictive values were given as $89 \%, 97 \%, 98 \%$ and $83 \%$, respectively. The diagnostic accuracy was $91 \%$. Additionally, the sensitivity of EBUS-TBNA in subtyping lymphomas high-grade non-Hodgkin lymphoma, low-grade non-Hodgkin lymphoma, and Hodgkin lymphoma was 90 \%, 100 $\%$, and $79 \%$, respectively. EBUS-TBNA diagnosis was adequate for clinical management in $84 \%$ of the patients. They concluded that EBUS-TBNA may be a good choice even in de novo lymphoma. However, when compared to other studies, it should be noted that the overall mean number of passes per lymph node was 5.2 (2-12). It may be translated as a high-volume specimen with relevant strategy to overcome the weakness of EBUS-TBNA in the diagnosis of lymphoma. The results of EBUS-TBNA was mostly consistent with final diagnoses; with only one patient originally diagnosed with a probable HL by means of EBUS-TBNA, who was later diagnosed with high-grade NHL. One of two inadequate samples were diagnosed as high-grade NHL and the other as granulomatous lymphadenitis (15). Related to the differences in retrospective design, the diagnostic performance of EBUS-TBNA was found in a wider range in other studies. $(13,16,17)$ This is true for the negative predictive value which has reached $100 \%$. However, regarding the predefined lymphoma suspicion, an overall negative predictive value of about $86.0 \%$ may be more realistic (16).

A literature review shows that EBUS-TBNA may be an option in the diagnosis of lymphoma (12). However, before the conclusion, several points need to be addressed. First, a predefined suspicion for lymphoma should be considered when EBUS-TBNA is being planned. As discussed earlier, for the presence of ROSE, needle selection and number of needle passes per lesion may be important. Secondly, cytological diagnosis of HL is very difficult. Classical Hodgkin's lymphoma is characterized by the presence of CD30 $0^{+}$ $\mathrm{CD} 15^{+} \mathrm{CD} 20^{+/-} \mathrm{CD} 45^{-}$Hodgkin and Reed-Sternberg (HRS) cells (5). They are scarce throughout the lymph node between dense fibrous bands and thick cellular background of lymphocytes, plasma cells and eosinophils. They also may co-exist with sarcoid-like granulomas. Hence, the diagnosis may be problematic in some cases $(5,30,31)$. This is also true for some NHL subtypes. The presence of necrosis and geographic involvement of lymph nodes may affect the results ofEBUS-TBNA $(3,4)$. Hence, lymph nodes/masses should be sampled in different areas and also different lymph nodes should be punctured in a particular station. Thirdly, EBUS-TBNA may be a choice in patients with the history of mediastinoscopy. Although it is accepted as a safe and efficient procedure, repeated mediastinoscopy is related to exposure problems and degree of morbidity, as there may be extensive fibrosis and adhesions (32, 33). Lastly, the diagnostic performance of EBUS-TBNA seems to be acceptable in the diagnosis of NHL and especially relapsed disease. The sensitivity had reached $100 \%$ in different studies. The cytologist should be fully informed on the previous history of the patient. When a higher possibility of NHL in elder patients with high SUVmax values is considered, EBUS-TBNA may be a choice without remarkable complication rate in elderly. There was no reported complication related to EBUS-TBNA operation in the studies related to the diagnosis of lymphoma (34). This is consistent with low complication rate in all indications. We should accept that EBUS-TBNA is a safe diagnostic tool; the complication possibility should not be considered to be a factor in the diagnosis of lymphoma.

The main strength of this paper is giving a surgical confirmation for all negative lesions. However, there are several weaknesses. This is a retrospective analysis with a relatively low number of lymphoma cases. Although the number of needle passes was acceptable we did not have ROSE and also our center lacked multidirectional modalities such as flow cytometry (10). We have used only 22-gauge needles routinely, which may be replaced by newer choices in the future. We do not have any information on the follow-up of the patients who had been diagnosed with EBUS, whether oncologists consulted the surgeons to obtain a surgical biopsy for confirmation or subtyping or no (7). And lastly, almost all of the papers agreed with a positive result being a "true positive". However, Farmer et al clearly showed and warned about possible "false positives" (9). These points should be addressed in the future studies.

In conclusion, it is very difficult to establish a suggestion as most of the evidence in this subject is derived from retrospective non-randomized studies with a limited number of patients. However, EBUS-TBNA may be preferred as an initial diagnostic modality in patients suspicious for lymphoma, recurrent disease and NHL type instead of repeated mediastinoscopy. Needle types may be changed in these patients and needle passes should be increased if there is no ROSE. Different lymph nodes in a particular station should be sampled as well as different areas of an individual lymph node or mass. Multidirectional analyses such as histomorphology, 
immunohistochemistry, flow cytometry and FISH should be conducted. And lastly, in patients in whom EBUS-TBNA failed, surgical biopsy would be appropriate rather than a repeated procedure.

\section{References}

1. Duwe BV SD, Musani AI. Tumors of the mediastinum. Chest 2005; 128 (4): 2893-909.

2. Swerdlow SH CE, Harris NL et al. WHO classification of tumours of haematopoietic and lymphoid tissues. In: Bosman FT, Jaffe ES, Lakhani SR, Ohgaki H, eds. World Health Organization Classification of Tumours. Lyon, France: IARC; 2008.

3. Dreyling M, Geisler C, Hermine O, Kluin-Nelemans HC, Le Gouill S, Rule $\mathbf{S}$ et al. Newly diagnosed and relapsed mantle cell lymphoma: ESMO Clinical Practice Guidelines for diagnosis, treatment and follow-up. Ann Oncol 2014; 25 (Suppl 3): iiir3-92.

4. Dreyling M, Ghielmini M, Rule S, Salles G, Vitolo U, Ladetto M et al. Newly diagnosed and relapsed follicular lymphoma: ESMO Clinical Practice Guidelines for diagnosis, treatment and follow-up. Ann Oncol 2016; 27 (Suppl 5): v83-v90.

5. Eichenauer DA, Engert A, Andre M, Federico M, Illidge T, Hutchings $\mathbf{M}$ et al. Hodgkin's lymphoma: ESMO Clinical Practice Guidelines for diagnosis, treatment and follow-up. Ann Oncol 2014; 25 Suppl 3: iii70-75.

6. Du Rand IA BP, Goldring J et al. British Thoracic Society Interventional Bronchoscopy Guideline Group. British Thoracic Society guideline for advanced diagnostic and therapeutic flexible bronchoscopy in adults. Thorax 2011; 66 (Suppl 3): iii1-iii21.

7. Kennedy MP JC, Bruzzi JF et al. Endobronchial ultrasound-guided transbronchial needle aspiration in the diagnosis of lymphoma. Thorax 2008; 63 (4): 360-365.

8. Bandyopadhyay D PT, Mehta AC. EBUS-TBNA for the Diagnosis of Lymphoma. Still an Achilles Heel. Ann Am Thorac Soc 2015; 12 (9): 1263-1264.

9. Farmer PL BD, Burns BF et al. The reliability of lymphoma diagnosis in small tissue samples is heavily influenced by lymphoma subtype. Am J Clin Pathol 2007; 128 (3): 474-480.

10. Gindesgaard CB SL, Christensen RK. Endobronchial ultrasound-guided transbronchial needle aspiration in an unselected cohort. J Bronchol Interv Pulm 2013; 20: 140-146.

11. Alici IO DN, Yilmaz A et al. The combination of cytological smears and cell blocks on endobronchial ultrasound-guided transbronchial needle aspirates allows a higher diagnostic yield. Virchows Arch 2013; 462 (3): 323-327.

12. Sharma D LZ, Dotson T, Bellinger CR. The Role of Endobronchial Ultrasound-guided Transbronchial Needle Aspiration in the Diagnosis and the Subtyping of Lymphoma in the Mediastinum. Clin Pulm Med 2016; 23 (3): $145-147$.

13. Erer OF ES, Anar C et al. Diagnostic yield of EBUS-TBNA for lymphoma and review of the literature. Endosc Ultrasound 2016.

14. Korrungruang P OM, Saka $\boldsymbol{H}$ et al. Endobronchial ultrasound-guided transbronchial needle aspiration is useful as an initial procedure for the diagnosis of lymphoma. Respir Investig 2016; 54 (1): 29-34.

15. Moonim MT BR, Fields PA, Santis G. Diagnosis and subtyping of de novo and relapsed mediastinal lymphomas by endobronchial ultrasound needle aspiration. Am J Respir Crit Care Med 2013; 188 (10): 1216-1223.

16. Grosu HB IM, Caraway NP et al. Endobronchial Ultrasound-Guided Transbronchial Needle Aspiration for the Diagnosis and Subtyping of Lymphoma. Ann Am Thorac Soc 2015; 12 (9): 1336-1344.
17. Iqbal S DZ, Kurtin PJ et al. Endobronchial ultrasound and lymphoproliferative disorders: a retrospective study. Ann Thorac Surg 2012; 94 (6): 1830-1834.

18. Marshall CB JB, Patel $\mathbf{S}$ et al. The utility of endobronchial ultrasoundguided transbronchial needle aspiration biopsy in the diagnosis of mediastinal lymphoproliferative disorders. Cancer Cytopathol 2011; 119 (2): 118-126.

19. Wahidi MM HF, Yasufuku K et al. Technical Aspects of Endobronchial Ultrasound Guided Transbronchial Needle Aspiration: CHEST Guideline and Expert Panel Report. Chest 2016; 149 (3): 816-835.

20. Nason KS KA, Schuchert MJ et al. Endobronchial Ultrasound-Transbronchial Needle Aspiration for Lymphoma in Patients With Low Suspicion for Lung Cancer and Mediastinal Lymphadenopathy. Ann Thorac Surg 2016; 101 (5): 1856-1863.

21. Senturk A BE, Kilic $\mathbf{H}$ et al. Endobronchial Ultrasound-Guided Transbronchial Needle Aspiration in the Diagnosis of Lymphoma. Asian Pacific Journal of Cancer Prevention 2014; 15 (10): 4169-4173.

22. Oki M SH, Kitagawa $C$ et al. Randomized Study of 21-gauge Versus 22-gauge Endobronchial Ultrasound-guided Transbronchial Needle Aspiration Needles for Sampling Histology Specimens. J Bronchol Intervent Pulmonol 2011; 18: 306-310.

23. Nakajima T, Yasufuku K, Takahashi R, Shingyoji M, Hirata T, Itami $\mathbf{M}$ et al. Comparison of 21-gauge and 22-gauge aspiration needle during endobronchial ultrasound-guided transbronchial needle aspiration. Respirology 2011; 16 (1): 90-94.

24. Inoue M NT, Tsujimura $\mathbf{H}$ et al. Mediastinal Follicular Lymphoma Diagnosed with Multidirectional Analysis Using Tissue Samples Obtained by EBUS-TBNA. Intern Med 2010; 49 (19): 2147-2149.

25. Ariza-Prota MAAA, Perez L et al. From cytology to histology: diagnosis of a relapsed mediastinal lymphoma by endobronchial ultrasound transbronchial histological needle. Respir Case Rep 2015; 3 (2): 68-71.

26. Witt BL, Adler DG, Hilden K, Layfield LJ. A comparative needle study: EUS-FNA procedures using the HD ProCore ${ }^{\mathrm{TM}}$ and EchoTip ${ }^{\circledR} 22$-gauge needle types. Diagnostic Cytopathology 2013; 41 (12): 1069-1074.

27. Furukawa BS BM, Siddiqi N, Pastis NJ. Diagnosing Hodgkin Lymphoma From an Endobronchial Ultrasound Core Needle Biopsy. J Bronchol Interv Pulmonol 2016; 23 (4): 336-339.

28. Creemers $\mathrm{K}$ vdHO, Los $\mathbf{J}$ et al. Endoscopic ultrasound fine needle aspiration in the diagnosis of lymphoma. J Oncol 2011; 2011: 785425.

29. Steinfort DP CM, Tsui A et al. Endobronchial Ultrasound-Guided Transbronchial Needle Aspiration for the Evaluation of Suspected Lymphoma. J Thorac Oncol 2010; 5: 804-809.

30. Xavier de Carvalho A, Maiato H, Maia AF, Ribeiro SA, Pontes P, Bickmore $\mathbf{W}$ et al. Reed-Sternberg cells form by abscission failure in the presence of functional Aurora B kinase. PLoS One 2015; 10 (5): e0124629.

31. Sakai J TK, Takahashi N et al. Lymphocyte-Rich Classical Hodgkin Lymphoma: A Case with Difficulty in Distinguishing from Nodular LymphocytePredominant Hodgkin Lymphoma. J Clin Exp Hematop 2015; 55 (1): 23-28.

32. Meersschaut D VF, Brutel de la Riviere A et al. Repeat Mediastinoscopy in the Assessment of New and Recurrent Lung Neoplasm. Ann Thorac Surg 1992; 53: 120-122.

33. Call S, Rami-Porta R, Obiols C, Serra-Mitjans M, Gonzalez-Pont G, Bastus-Piulats R et al. Repeat mediastinoscopy in all its indications: experience with 96 patients and 101 procedures. Eur J Cardiothorac Surg 2011; 39 (6): 1022-1027.

34. Kheir F IA, Assasa O, Alraiyes AH. The utility of endobronchial ultrasound-transbronchial needle aspiration in lymphoma. Endosc Ultrasound 2016; 5 (1): 43-48.

Received February 18, 2018. Accepted March 23, 2018. 